\title{
CONCOMITANT URINARY TRACT INFECTIONS IN RETROGRADE INTRARENAL SURGERY (RIRS)
}

\author{
Ivan Velev ${ }^{1}$, Deyan Anakievski ${ }^{2}$ \\ ${ }^{1}$ Department of Urology, Sofiamed University Hospital, Sofia \\ ${ }^{2}$ Clinic of Urology, St. Marina University Hospital, Medical University of Varna
}

\begin{abstract}
INTRODUCTION: Urinary stones affect approximately 5 to $15 \%$ of the population in developed countries and depend on geographical location, age and gender. Laser lithotripsy has already become established worldwide as a preferred method for their treatment in urological practice. At present, retrograde intrarenal surgery (RIRS) has shown a high success rate and an acceptable number of complications. Recent studies show a complication rate of 9 to $25 \%$ of ureteroscopic procedures in different parts of the world. Urinary tract infections, ureteral lesions, hematuria, and postoperative renal colic are the most common complications in this type of surgery.
\end{abstract}

AIM: The main goal of our study is to analyze the characteristics and risk factors of complicated urinary tract infections during retrograde intrarenal surgery and to look for the most effective options for their prevention and treatment.

MATERIALS AND METHODS: The study was conducted in two university hospitals in Varna and Sofia under relatively similar conditions and equipment. Between January 2019 and June 2020, this study retrospectively included 324 patients who underwent RIRS for the treatment of kidney stones with 212 patients undergoing laser treatment at the Clinic of Urology at St. Marina University Hospital, Varna, and 112 patients-in the Urology Department at Sofiamed University Hospital, Sofia.

RESULTS: The overall incidence of postoperative urinary tract infections was $14.5 \%$ (47/324 patients). The mean age of the patients was $52.8 \pm 13.1$ years, out of 324 patients, 217 patients (67\%) were men and 107 patients (33\%) were women. Major diseases included diabetes mellitus-52 (16\%), and chronic kidney disease 16 (4.9\%).

CONCLUSION: Despite adequate prophylactic measures, postoperative urinary tract infections after ureteroscopy (URS) were found in $\mathbf{1 4 . 5 \%}$ of the cases of this study and the most commonly found microorganism, which was isolated in more than half of patients with febrile urinary tract infection, was E. coli.

In addition, it is necessary to reduce the operative working time, because this is the most important factor that prevents postoperative urinary tract infection.

Address for correspondence:

Keywords: laser lithotripsy, flexible ureteroscope,

Deyan Anakievski

1 Hristo Smirnenski Blvd

Clinic of Urology

St. Marina University Hospital

9010 Varna, Bulgaria

e-mail:dejan_anakievski@yahoo.com

Received: February 27, 2021

Accepted: March 19, 2021 


\section{INTRODUCTION}

Urinary stones affect approximately 5 to $15 \%$ of the population in developed countries and depend on geographical location, age and gender. Laser lithotripsy has already become established worldwide as a preferred method for their treatment in urological practice. The first flexible ureteroscopic procedures were introduced around the 1960s and 1970s, when Marshall (1) described an endoscopic examination of the upper urinary tract in 1964, and in 1971 Takayasu (2) performed the first flexible endoscopy and published it. Thanks to the creation of ureterorenoscopes with a smaller diameter and greater flexibility, almost 20 years later, in 1987, Bagley $(3,4)$ gave the first guidelines with which flexible ureteroscopy has become one of the standard procedures today to remove small or medium-sized kidney stones. At present, retrograde intrarenal surgery (RIRS) has shown a high success rate and an acceptable number of complications (4). Recent studies show a complication rate of 9 to $25 \%$ of ureteroscopic procedures in different parts of the world (5-9). Urinary tract infections, ureteral lesions, hematuria, and postoperative renal colic are the most common complications in this type of surgery $(10,11)$. The object of increased attention is the increased risk of urinary tract infections, which occurs in 1 to $7 \%$ of all ureteroscopies. To prevent concomitant infections during the procedure, antibiotics are used prophylactically on a regular basis, as fluoroquinolones have traditionally been the most commonly used $(12,13)$. However, postoperative febrile urinary tract infections are not uncommon and occur even with perioperative antibiotic prophylaxis. The most common causes and factors that are significantly associated with postoperative fever and risk of sepsis are: the presence of a preoperative stent, obstructive pyelonephritis, a positive result of preoperative bladder culture, female gender, larger stone size, and prolonged operative time (14).

\section{AIM}

The main goal of our study is to analyze the characteristics and risk factors of complicated urinary tract infections during retrograde intrarenal surgery and to look for the most effective options for their prevention and treatment.

\section{MATERIALS AND METHODS}

The study was conducted in two university hospitals in Varna and Sofia under relatively similar conditions and equipment. Between January 2019 and June 2020, this study retrospectively included 324 patients who underwent RIRS for the treatment of kidney stones with 212 patients undergoing laser treatment at the Clinic of Urology at St. Marina University Hospital, Varna and 112 patients-in the Urology Department at Sofiamed University Hospital, Sofia. Perioperative data included in the retrospective analysis contained age, sex, underlying diseases (diabetes mellitus, chronic renal failure), presence of hydronephrosis, preoperative stent, preoperative percutaneous nephrostomy, previous lithotripsy, number and size of stones. Preoperative urocultures were performed and patients with preoperative urinary tract infection (pyuria or bacteriuria) were excluded from this study. Third-generation cephalosporins were administered prophylactically in the morning before surgery, and the oral form was administered for 5 to 10 days postoperatively after discharge. Postoperative urinary tract infection is defined by the appearance of fever above $38^{\circ} \mathrm{C}$ combined with bacteriuria within 1 week of surgery and without infectious signs in other organs. The frequency and causes of positive uroculture were reported.

\section{TECHNIQUE}

Flexible ureteroscopes by Karl Storz and Olympus were used for surgical interventions in a small number of cases and mainly a single one by Pusen. The patient is placed in a free lithotomy position to facilitate the insertion and easy passage of the protective shaft and ureteroscope in the most straightforward manner possible. After cystoscopy, a hydrophilic guidewire is inserted into the kidney, the ureter is visually and fluoroscopically monitored vertically with a semi-rigid ureteroscope, and a second guidewire is placed. In most cases, a $12 / 14 \mathrm{Fr}$ protective shaft is placed on it, which not only helps to improve visibility during the operation, but also to maintain a low internal pressure and facilitates the extraction of fragments. We usually use a gravity irrigation system in combination with a manual increase in the pressure of the washing liquid by $50 \mathrm{~mL}$ syringe. The 200-272 $\mu \mathrm{m}$ laser fibers we use allow all degrees of flexion of the ureteroscope, performing 
in situ lithotripsy in cup concretions and achieving a high degree of disintegration. In the case of particularly hard stones, we prefer to perform fragmentation only as much as necessary to extract as many fragments as possible and to leave some residual particles for spontaneous elimination. In cases of soft stones, fragments up to 2-3 mm can be left after in situ lithotripsy, which will be eliminated spontaneously during the postoperative period, thus shortening the working time. At the end of the procedure, a ureteral stent is placed in almost all patients.

\section{RESULTS}

The overall incidence of postoperative urinary tract infections was $14.5 \%$ (47/324 patients). Table 1 shows the demographic and clinical charac-

Table 1. Demographic and clinical characteristics of the patients

\section{Patient Characteristics}

\begin{tabular}{lc} 
Number of patients & 324 \\
Average age & 52.8 \\
Gender: & \\
$\quad$ Men & $217(67 \%)$ \\
Women & $107(33 \%)$ \\
Risk factors: & \\
Diabetes mellitus & $52(16 \%)$ \\
Chronic renal failure & $16(4.9 \%)$ \\
Nephrostomy & $23(7 \%)$ \\
Ureteral stent & $55(17 \%)$ \\
Previous lithotripsy & $68(21 \%)$ \\
Hydronephrosis & $148(45.6 \%)$ \\
\hline \hline
\end{tabular}

teristics of the patients. The mean age of the patient was $52.8 \pm 13.1$ years, out of 324 patients, 217 patients (67\%) were men and 107 patients (33\%) were women. Major diseases included diabetes mellitus 52 (16\%) and chronic kidney disease 16 (4.9\%). A total of 68 patients $(21 \%)$ had a history of previous lithotripsy and 148 patients $(45.6 \%)$ had preoperative hydronephrosis. A total of 23 patients (7.0\%) had percutaneous nephrostomies and 55 patients (18.1\%) had preoperative urethral stents. The average operating time was $47.1 \pm 27.9$ minutes with an average stone size of $10.0 \pm 4.1 \mathrm{~mm}$.

Table 2 shows the comparison of the clinical information between the group of postoperative urinary tract infections and the non-febrile group.

The size of the stone and the working time are significantly different between the group of febrile postoperative urinary tract infections and the nonfebrile group.

Table 3 shows the results in the two clinics. There was no significant difference in the frequency of postoperative urinary tract infections compared to the number of operated patients in the different clinics. Out of 47 patients with febrile urinary tract infections, pathogenic microorganisms were cultured in the urine of 32 patients (68\%), in 15 patients (32\%) they were not detected. Escherichia coli was the most commonly cultivated bacterial agent in a total of 24 patients (51\%).

In all 47 patients with fever, 3rd generation cephalosporins were initially administered while pathogenic microorganisms were cultured. Of the 32 patients with a pathogen identified in their serum or uroculture, antibiotic therapy was changed

Table 2. Comparison of the clinical information between the group of postoperative urinary tract infections and the non-

\begin{tabular}{lcc} 
Postoperative Febrile UTI & Yes & No \\
Number of patients & $47(14.5 \%)$ & $277(85.4 \%)$ \\
Gender: Men/Women & $35(74.5 \%) / 12(25.5 \%)$ & \\
Diabetes mellitus & $13(30.2)$ & $39(13.9)$ \\
Chronic renal failure & $4(9.3)$ & $12(4.3)$ \\
Previous lithotripsy & $9(20.9)$ & $39(13.9)$ \\
Nephrostomy & $10(2.3)$ & $13(0.7)$ \\
Ureteral stent & $8(18.6)$ & $47(16.7)$ \\
Hydronephrosis & $20(46.5)$ & $128(45.6)$ \\
Size of concrement $(\mathrm{mm})$ & 13.3 & 9.79 \\
Operative time (min) & 62.77 & 44.54 \\
\hline
\end{tabular}


Table 3. Results in the two clinics

\begin{tabular}{lcc|}
\hline \multirow{2}{*}{ Results } & \multicolumn{2}{c}{ Results by Clinic } \\
& Sofia & Varna \\
\hline Number of patients & 112 & 212 \\
Postoperative febrile UTI & 16 & 31 \\
Proven by microbiology & $14.2 \%)$ & $(14.6 \%)$ \\
Unproven & 4 & 20 \\
Escherichia coli & 8 & 11 \\
Enterococcus faecalis & 3 & 16 \\
Pseudomonas aeruginosa & 1 & 2 \\
Proteus mirabilis & 0 & 1 \\
Klebsiella & 0 & 1 \\
\hline \hline
\end{tabular}

to aminoglycosides and/or ampicillin/sulbactam in 17 patients, vancomycin in 5 patients, and meropenem in 10 patients. Of the 15 patients without an identified pathogen in uroculture, 9 were treated with cefoperazone/sulbactam and 6 patients were on aminoglycosides.

\section{DISCUSSION}

Fluoroquinolones have traditionally been used for antibiotic prophylaxis for the past 20 years and have been recommended as the first choice, but their overuse and abuse have led to an increased resistance to them. In 2018 the European Medicines Agency (EMA) strongly restricted the use of two groups of antibiotics, quinolones and fluoroquinolones, for this reason, as well as the increasing number of longlasting side effects. This was the motivation behind our means of choice as initial empirical treatment, which was the use of 3rd generation cephalosporins, after which the antibiotic was adjusted according to the results obtained from uroculture. In cases where the bacterial causative agent was not isolated, but there was a rapid response to the febrile condition, normalization of clinical and laboratory parameters such as leukocytes, C-reactive protein, pyuria, the drug was not changed until the patient was discharged.

The resistance of pathogenic microorganisms to antibiotics was similar in each center. In our case, the most common pathogen was Escherichia coli, which was identified in 24 patients (51\%). Bacteriuria, previous catheterization, preoperative ureteral stents, and percutaneous nephrostomy are risk factors for febrile complications. Cases of preoperative stent and percutaneous nephrostomy were observed for urinary tract infection and rehabilitated prior to hospitalization in both centers.

In our study, we report that operative time is a major risk factor for postoperative urinary tract infections due to prolonged irrigation of lavage fluid and is one of the reasons for the higher incidence of urinary tract infections. Long operating time with prolonged irrigation is one of the reasons for their high frequency. Irrigation rate and volume have been shown in the past to be independent risk factors for urinary tract infections after ureteroscopy. The high pressure in the pyelocalyx system and the reflux systemic absorption of lavage fluid containing bacteria or toxins have been shown to contribute to the incidence of postoperative infections after ureteroscopy (URS) $(15,16)$. If the size of the stone is large, the operation time may be longer, but the size of the stone is not significantly correlated with the frequency of febrile urinary tract infections. The composition (hardness) of the stones, not the size, is the reason for the longer working time.

\section{CONCLUSION}

Despite adequate prophylactic measures, postoperative urinary tract infections after URS were found in $14.5 \%$ of the cases of this study and the most commonly found microorganism, which was isolated in more than half of patients with febrile urinary tract infection, was E. coli. Due to the fact that most of the microorganisms in our practice in recent years are resistant to fluoroquinolones, which are regularly used as prophylactic antibiotics, postoperative prophylaxis was carried out with 2-3 generations of cephalosporins. Although not all urocultures were positive, in cases of clinically and biochemically manifested postoperative urinary tract infection, more powerful antibiotic therapy was used to control it. In addition, it is necessary to reduce the operative working time, because this is the most important factor that prevents postoperative urinary tract infection. 


\section{REFERENCES}

1. Marshall VF. Fiber optics in urology. J Urol. 1964; 91:110-4. doi: 10.1016/s0022-5347(17)64066-7.

2. Takayasu H, Aso Y, Takagi T, Go T. Clinical application of fiber-optic pyeloureteroscope. Urol Int. 1971; 26(2):97-104. doi: 10.1159/000279719.

3. Bagley DH, Huffman JL y Lyon ES. Flexible ureteropyeloscopy: diagnosis and treatment in the upper urinary tract. J Urol. 1987; 138(2):280-5. doi: 10.1016/s0022-5347(17)43119-3.

4. Bagley DH, Rittenberg MH. Intrarenal dimensions. Guidelines for flexible ureteropyeloscopes. Surg Endosc 1987; 1(2):119-21. doi: 10.1007/BF00312699. PMID: 3503366.

5. Stav K, Cooper A, Zisman A, Leibovici D, Lindner A, Siegel YI. Retrograde intrarenal lithotripsy outcome after failure of shock wave lithotripsy. J Urol. 2003; 170(6 Pt 1):2198-201. doi: 10.1097/01. ju.0000096421.56225.79.

6. Fabrizio MD, Behari A, Bagley DH. Ureteroscopic management of intrarenal calculi. J Urol. 1998; 159(4):1139-43.

7. Volkin D, Shah O. Complications of ureteroscopy for stone disease. Minerva Urol Nefrol. 2016; 68(6):570-85.

8. Geavlete P, Georgescu D, Niţă G, Mirciulescu V, Cauni V. Complications of 2735 retrograde semirigid ureteroscopy procedures: a single-center experience. J Endourol. 2006; 20(3):179-85. doi: 10.1089/end.2006.20.179.

9. Skolarikos A, Mitsogiannis H, Deliveliotis C. Indications, prediction of success and methods to improve outcome of shock wave lithotripsy of renal and upper ureteral calculi. Arch Ital Urol Androl. 2010; 82(1):56-63.
10. Breda A, Ogunyemi O, Leppert JT, Schulam PG. Flexible ureteroscopy and laser lithotripsy for multiple unilateral intrarenal stones. Eur Urol. 2009; 55(5):1190-6. doi: 10.1016/j.eururo.2008.06.019.

11. Cindolo L, Castellan P, Scoffone CM, Cracco CM, Celia A, Paccaduscio A, et al. Mortality and flexible ureteroscopy: analysis of six cases. World J Urol. 2016; 34(3):305-10. doi: 10.1007/s00345-015-1642-0.

12. Wolf JS, Bennett CJ, Dmochowski RR, Hollenbeck BK, Pearle MS, Schaeffer AJ. Best practice policy statement on urologic surgery antimicrobial prophylaxis. J Urol. 2008; 179(4):1379-90. doi: 10.1016/j. juro.2008.01.068.

13. Knopf HJ, Graff HJ, Schulze H. Perioperative antibiotic prophylaxis in ureteroscopic stone removal. Eur Urol. 2003; 44(1):115-8. doi: 10.1016/ s0302-2838(03)00189-1.

14. Sohn DW, Kim SW, Hong CG, Yoon BI, Ha US, Cho YH. Risk factors of infectious complication after ureteroscopic Infectious complications after URS procedures of the upper urinary tract. J Infect Chemother. 2013; 19(6):1102-8. doi:10.1007/ s10156-013-0632-7.

15. Osther PJS. Risks of flexible ureterorenoscopy: pathophysiology and prevention. Urolithiasis. 2018; 46(1):59-67. doi: 10.1007/s00240-017-1018-6.

16. Osther PJ, Pedersen KV, Lildal SK, Pless MS, Andreassen KH, Osther SS, et al. Pathophysiological aspects of ureterorenoscopic management of upper urinary tract calculi. Curr Opin Urol. 2016; 26(1):63-9. doi: 10.1097/MOU.0000000000000235. 